\title{
THE ASSOCIATION OF TREE SPECIES DIVERSITY AND ABUNDANCE WITH THE SOIL EDAPHIC FACTOR IN A LARGEST TROPICAL RECREATIONAL FOREST OF TERENGGANU, PENINSULAR MALAYSIA
}

\author{
KHAIRIL MAHMUD ${ }^{1 *}$, KHAIRULAKWA, H. ${ }^{2}$, NUR FATIHAH, H.N. ${ }^{2}$, \\ NORNASUHA, Y. ${ }^{2}$, KHANDAKER, M.M. ${ }^{2}$, MOHD IZUAN EFFENDI HALMI ${ }^{3}$, \\ NOOR-AMALINA, R. ${ }^{4}$ and WAN JULIANA, W.A. ${ }^{5,6}$ \\ ${ }^{1}$ Department of Crop Science, Faculty of Agriculture, Universiti Putra Malaysia (UPM), \\ 43400 Seri Kembangan, Selangor, Malaysia \\ ${ }^{2}$ Faculty of Bioresources and Food Industry, Universiti Sultan Zainal Abidin (UniSZA), \\ Tembila Campus, 22200 Besut, Terengganu, Malaysia \\ ${ }^{3}$ Department of Land Management, Faculty of Agriculture, Universiti Putra Malaysia (UPM), \\ 43400 Seri Kembangan, Selangor, Malaysia \\ ${ }^{4}$ Faculty of Health Science, Universiti Kebangsaan Malaysia (UKM), \\ Jalan Raja Muda Abdul Aziz, 50300 Kuala Lumpur, Malaysia \\ ${ }^{5}$ Department of Earth Sciences and Environment, Faculty of Science and Technology, \\ Universiti Kebangsaan Malaysia (UKM), 43600 Bangi, Selangor, Malaysia \\ ${ }^{6}$ Institute of Climate Change, Universiti Kebangsaan Malaysia (UKM), \\ 43600 Bangi, Selangor, Malaysia \\ *E-mail: khairilmahmud@upm.edu.my
}

Accepted 12 June 2020, Published online 30 June 2020

\begin{abstract}
A study was conducted to investigate the association between tree species composition with soil edaphic factor in Chemerong Recreational Forest, the largest recreational forest in Terengganu, Peninsular Malaysia. Two types of forest were chosen which are riparian forest and inland forest. Four plots with the dimension of $50 \mathrm{~m} \times 20 \mathrm{~m}$ each were established with two plots at each forest type with total study site of 0.4 ha. A total of 1158 trees ( $\geq 1 \mathrm{~cm}$ diameter) from 263 species, 125 genus and 50 families were recorded. The higher species number was recorded in the inland forest with 175 species, 103 genus and 45 families compared to riparian plot with 154 species, 109 genera and 39 families. Lijndenia laurina was found to be the important species in the riparian forest with Important Value Index (IVi) of 5.22\% while Mangifera caesia at the inland forest with $3.21 \%$. The Shannon-Weiner diversity indexes $\left(H^{\prime}\right)$ was considered high in all two types of forest with 5.04 at the riparian forest and 5.14 at the inland forest. Sorenson's community similarity coefficient (CCs) showed the tree species communities, between the two types of forest had low similarities with 0.38. A total 33 endemic species in Peninsular Malaysia were found at Chemerong Recreational Forest. Fifty-five species in this study were listed in the International Union for Conservation of Nature IUCN red list of threatened species 2019. Significant differences were found in phosphorus, electric conductivity, ammonium nitrate, moisture content and organic matter between these forests. Canonical Correspondence Analysis (CCA) showed less association between species composition with the physico-chemical characyeristics of soil in this study indicating the soil edaphic factor is not the main factor controlling the species distribution at this site.
\end{abstract}

Key words: Riparian forest, soil chemistry, Canonical Correspondence Analysis (CCA), recreational forest, Peninsular Malaysia

\footnotetext{
* To whom correspondence should be addressed.
} 


\section{INTRODUCTION}

Malaysia is one of the world's Mega Diversity countries and has an estimated 20.62 million hectares of natural forests in 2012 , covering $62.5 \%$ of the country's land area (UNDP, 2017). Malaysia has over 150 major rivers; as well a variety of tropical wetlands, forest, coastal and marine ecosystems, representing several Global 200 Eco regions, and it is recognized as one of $17 \mathrm{mega}-$ diverse countries in the world (UNDP, 2017). Peninsular Malaysia has about 13.68 million hectares land area and about 5.77 million hectares are forested area around $85.29 \%$ (Forestry Department Peninsular Malaysia, 2016). Forested area has 4.92 million hectare permanent reserved forests, a 4.16 million hectare Inland Forest, 0.25 million hectares peat swamp forests, 0.11 million hectares Mangroves and 0.40 million hectares plantation forest (Forestry Department Peninsular Malaysia, 2016). The tropical rainforest in Peninsular Malaysia covers about 5.87 million hectares or $45 \%$ of its total land (UNDP, 2017). Dipterocarp forests account for about $85 \%$ of the country's forested areas and are commonly composed of species from the genera Anisoptera, Dipterocarpus, Dryobalanops, Hopea, Shorea and Parashorea (Ashton, 2008).

Terengganu state has rich and diverse biodiversity and their forest resources are seen to have the potential to generate the economy from horticultural, medicinal plants, timber and also as a tourist and recreational centre. Chemerong Recreational Forest is located in Pasir Raja Forest Reserve, which is a primary forest located in Dungun District, Terengganu, Peninsular Malaysia. It covers an area of 300 hectares and is located about $30 \mathrm{~km}$ from Al-Muktafi Billah Shah town and $100 \mathrm{~km}$ from the capital city of Terengganu, Kuala Terengganu. The recreational forest was first developed in 1993 and was the largest recreational forest in Terengganu (Forestry Department of Malaysia, 2006; 2008). In the vast Pasir Raja Forest Reserve bordering the Taman Negara, Malaysia's premier National Park, stands the world's oldest 1300 years old and largest Chengal tree (Neobalanocarpus heimii). In addition, the highest waterfall (305 m) lies in Pasir Raja Forest Reserve and the nearby Chemerong Recreational Forest. Nevertheless, the Gunung Berembun (Berembun Mountain), which is close to the recreational forest is one of the best places for hiking in Terengganu. As one of the tourist attractions sites in Terengganu, Chemerong Recreational Forest received more than 20,000 people each year and considered as one of the important recreational forests in Terengganu (Forestry Department of Malaysia, 2016). Although Pasir Raja was gazetted as a permanent forest reserve, however some parts of the area had been deforested and not far from the recreational forest, there was an oil palm plantation area. All the while these activities may affect the ecosystem and biota of this area.

Studies on the tree species distribution at different forest types of Malaysian tropical forest were done previously by many researchers (Proctor et al., 1983; Condit et al., 1999; Nizam et al., 2011; Khairil et al., 2011; Khairil et al., 2014a; 2014b). The tree species distribution of these forest types was influenced by few factors such as altitude (Proctor et al., 1983), soil types (Nizam et al., 2011; Khairil et al., 2014a; 2014b) and their location which are intact with water bodies such as stream, lake and river (Khairil et al., 2011; 2013; 2014b). According to Khairil et al. (2011) the riparian forest and seasonal flood forest had lower species diversity and composition compared to the inland forest at Chini watershed, the second largest natural lake in Peninsular Malaysia. The tree species community at some riparian area and seasonal flood area were influenced by the water as sometimes these areas were inundated, especially during the raining season (Khairil et al., 2011; Khairil et al., 2014a; 2014b). In addition, the gap area along the rivers and beside the lake may influence the occurrence of selected tree species in the riparian area (Teixeira et al., 2008).

Soil plays an important role in plant growth as it provides useful major and minor nutrient elements and mineral and this contributes to the occurrence and distribution of tree species of tropical forest (Deyn et al., 2004; Sukri et al., 2012; Baldeck et al., 2013; Khairil et al., 2014b; 2015). Studies on the relationship between tree species abundance and distribution with the soil physico-chemical characteristics in tropical forest in Malaysia were done by many previous researchers (Davies et al., 2005; Nizam et al., 2006; Katabuchi et al., 2012; Khairil et al., 2014a; 2015). Most of the studies only cover the trees with $\geq 5 \mathrm{~cm}$ diameter ( $\mathrm{dbh}$ ) and none of the studies mentioned cover the lower tree dbh size. To date, there was no detailed study conducted on the tree species composition and seedlings in Chemerong Recreational Forest. Nevertheless, tree species diversity and abundance of Terengganu are less documented compared to its neighbouring states, Kelantan and Terengganu. According to Memiaghe et al. (2016), small-diameter tree populations are also important to the demographic rates and nutrient cycling, thus in this study, we sampled the trees with the size of $1 \mathrm{~cm}$ diameter and above in this area. By this sampling, the important features of the tree species composition and structure can be determined. Furthermore, by analysing the soil physico-chemical characteristics, the association between species abundance with the soil edaphic 
factor in this area also can be determined. The study conducted was following these hypotheses:

1. The tree species diversity and abundance of the tree species are significantly different between the inland and riparian forest at Chemerong Recreational Forest.

2. When comparing the soil physico-chemical characteristics of riparian and inland forest, the elements such as $\mathrm{P}, \mathrm{Ca}, \mathrm{Mg}$, organic matter, electrical conductivity (EC) may significantly different between these forest types.

3. The distribution and composition of the tree species in Chemerong Recreational Forest may have associated with soil edaphic factors. In this study, soil edaphic factor can be an important factor in shaping these patterns.

\section{MATERIALS AND METHODS}

\section{Study site}

Dungun is a coastal district state of Terengganu and considered as the second largest district after Hulu Terengganu. District of Dungun can be divided into 11 mukim; Besul, Hulu Paka, Jengai, Jerangau, Kuala Abang, Kuala Dungun, Kuala Paka, Kumpal, Rasau, Sura and Pasir Raja (Forestry Department of Malaysia, 2016). Total population in the district of Dungun is 149,851 people and Kuala Dungun is the capital city of this district. The Chemerong Recreational Forest is located in Pasir Raja Forest Reserves, Dungun, Terengganu and the distance to Kuala Dungun town is almost $30 \mathrm{~km}$ while the distance to the capital city of Terengganu, Kuala Terengganu is around $100 \mathrm{~km}$ (Forestry Department of Malaysia, 2008) (Figure 1). The Chemerong Recreational Forest is the largest permanent recreational forest in Terengganu, Peninsular Malaysia with the size of 300 ha and was gazetted in 1960 under the control by the Terengganu Forestry Department (Forestry Department of Malaysia, 2016).

\section{Plot establishment}

Two forest types were recognized in Chemerong recreational forests which were riparian forest; the forest beside the river and inland forest which is approximately more than $200 \mathrm{~m}$ away from the river bank. Stratified sampling method was used to build the study plots at these two forest types. Four plots with the size of $50 \mathrm{~m}$ x $20 \mathrm{~m}$ ( $0.1 \mathrm{ha})$ were established in this study which two plots at the riparian forest $\left(05^{\circ} 49.424^{\prime} \mathrm{N}, 102^{\circ} 00.043^{\prime} \mathrm{E} \& 05^{\circ}\right.$ $\left.19.419^{\prime} \mathrm{N}, 103^{\circ} 00.197^{\prime} \mathrm{E}\right)$ and another two plots at the inland forest $\left(05^{\circ} 39.413^{\prime} \mathrm{N}, 102^{\circ} 59.989^{\prime} \mathrm{E}\right.$ $\left.\& 05^{\circ} 39.4141^{\prime} \mathrm{N}, 103^{\circ} 19.901^{\prime} \mathrm{E}\right)$ (Figure 1).

\section{Plants sampling}

All trees with the diameter $1 \mathrm{~cm}$ and above were selected and the leaves were collected for identification purpose. The process of identification conducted was based on $\mathrm{Ng}(1978 ; 1989)$ and Whitmore $(1972 ; 1973)$ and with the help of senior botanist from Universiti Malaysia Terengganu (UMT) and Universiti Kebangsaan Malaysia (UKM). The density, important value index (IVi), composition and diversity of the tree species were based on Magurran (1988) and Brower et al. (1997). The Sorenson's community similarity index was analysed to measure the degree of species similarity between the two types of forest using the BIODAP software following Magurran (2011).

\section{Soil sampling}

Five soil samples were collected from each plot and total up to 20 samples at the depths of 0 to $15 \mathrm{~cm}$ by using an auger. Each soil samples with approximately $200 \mathrm{~g}$ in weight were then sent to the Universiti Sultan Zainal Abidin (UniSZA) lab for air-drying. The roots, small stones and leaves were separated from the soil and were sieved through a $2 \mathrm{~mm}$ sieve while lump soils were crushed using agate tools. These samples were then analysed for their physico-chemical characteristics, which were soil particle size distribution, organic matter content, exchangeable acid cation Aluminium $\left(\mathrm{Al}^{+}\right)$and Hydrogen $\left(\mathrm{H}^{+}\right)$, exchangeable basic cation, cation exchange capacity (CEC), electrical (EC), as well as available nutrients of Phosphorus (P), Magnesium $(\mathrm{Mg})$ and Potassium $(\mathrm{K})$. The soil organic matter compounds were analysed based on the loss ignition technique following Black (1968). The $\mathrm{pH}$ of the soil was determined using a soil: water ratio of 1:2.5 (Rowell, 1994; Shamshuddin, 1981). The exchangeable acidic cations $\left(\mathrm{Al}^{+}\right.$and $\left.\mathrm{H}^{+}\right)$were measured in $1.0 \mathrm{M} \mathrm{KCl}$ extract by titration while exchangeable basic cations of Magnesium $\left(\mathrm{Mg}^{2+}\right)$ and Potassium $\left(\mathrm{K}^{+}\right)$were measured in $1.0 \mathrm{M}$ ammonium acetate extract by Atomic Absorption Spectrophotometry, (Perkin Elmer, AA analyst 100, Norwalk, USA) (Black, 1968; Shamshuddin, 1981). Cation exchange capacity was obtained by summation of acid and basic cations. Electrical conductivity was determined using saturated gypsum extract following Rowell (1994). Available nutrients of Phosphorus (P), Ammonium Nitrate $\left(\mathrm{NH}^{4+} \mathrm{NO}^{3-}\right)$ using flow injection auto-analyser (FIA star 5000 Analyser and FOSS TECATOR 5027 Sampler for the auto sampler, Sweden), available $\mathrm{Mg}$ and $\mathrm{K}$ were extracted using sulphuric acid and determined using an Atomic Absorption Spectrophotometer (FAAS) (Perkin Elmer, AA analyst 100, Norwalk, USA) (Rowell, 1994). 


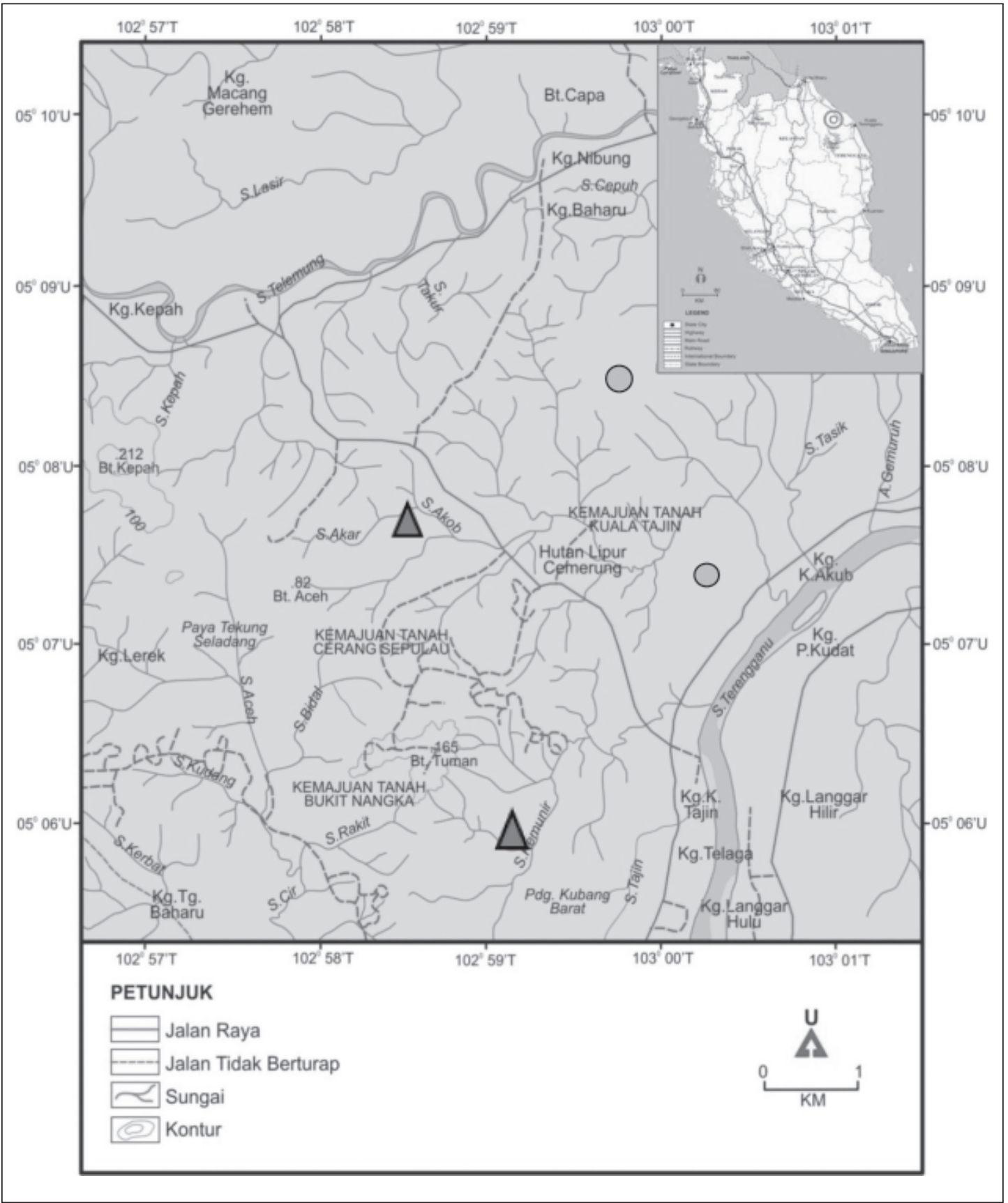

Fig. 1. The location of research plots at the Chemerong Recreational Forest, Dungun, Terengganu. Notes: $\triangle$ = riparian plot. $\bigcirc=$ inland plot. $(0)$ Study site location.

\section{Statistical Analysis}

Normality test and T-test was conducted to compare the mean of the soil parameter of two forest types by using the MINITAB 16 software. Canonical correspondence analysis (CCA) was conducted to investigate the patterns in tree species distribution in relation to edaphic variables by using the PCORD version 5.0 (McCune \& Grace, 2002; Baruch, 2005). The CCA method was used to illustrate the relationships between the two set factors (soil, $\mathrm{N}=$ 15 and trees $\mathrm{N}=263$ ). The occurrences of the species with less than 3 within the subplots were eliminated to ease the CCA analysis following Baruch (2005). Direct ordination of CCA examines the strength of floristic abundance with edaphic factors (Nizam et al., 2006; Khairil et al., 2014b).

\section{RESULTS AND DISCUSSION}

\section{Tree species composition and abundance}

A total of 1158 individual trees with $1 \mathrm{~cm}$ diameter were recorded in this study which consisted of 263 species, 125 genus and 50 families. The 
inland forest plot had higher individuals and species number with 636 individuals from 175 species, 103 genera and 39 families, whereas Riparian forest plot recorded 516 individuals from 154 species, 109 genera and 39 families (Table 1). In terms of class size, trees with the size of $1-4.99 \mathrm{~cm}$ dbh had the highest composition with 2335 ind/ha at inland forest plot and $1800 \mathrm{ind} / \mathrm{ha}$ at riparian forest plot
(Table 2). Lijndenia laurina (Melastomataceae) had the highest density of the riparian forest with 200 individuals (ind)/ hectare (ha) followed by Shorea macroptera (Dipterocarpaceae) with $120 \mathrm{ind} /$ ha and Gaertnera vaginans with 95 ind/ha. Meanwhile Streblus elongatus (Moraceae) was the most dense species in the inland forest plot with $140 \mathrm{ind} / \mathrm{ha}$ followed by Gluta elegans and Mangifera caesia

Table 1. Taxonomic composition of tree species at Chemerong Recreational Forest, Pasir Raja Forest Reserve, Terengganu, Malaysia

\begin{tabular}{|c|c|c|c|c|c|c|c|}
\hline \multirow{2}{*}{ Number } & \multirow{2}{*}{ Family } & \multicolumn{3}{|c|}{ Riparian } & \multicolumn{3}{|c|}{ Inland } \\
\hline & & Genera & Species & Ind & Genera & Species & Ind \\
\hline 1 & Alangiaceae & nil & nil & nil & 1 & 1 & 1 \\
\hline 2 & Anacardiaceae & 2 & 3 & 7 & 4 & 6 & 10 \\
\hline 3 & Anisophylleaceae & 1 & 2 & 5 & 1 & 3 & 35 \\
\hline 4 & Annonaceae & 7 & 8 & 29 & 8 & 13 & 36 \\
\hline 5 & Apocynaceae & 3 & 3 & 3 & nil & nil & nil \\
\hline 6 & Bombacaceae & 1 & 1 & 1 & 4 & 6 & 15 \\
\hline 7 & Burseraceae & 3 & 5 & 19 & nil & nil & nil \\
\hline 8 & Celastraceae & 1 & 1 & 2 & 2 & 2 & 11 \\
\hline 9 & Chrysobalanaceae & 1 & 1 & 1 & 1 & 1 & 1 \\
\hline 10 & Ctenolophonaceae & 1 & 1 & 3 & 1 & 1 & 3 \\
\hline 11 & Dilleniaceae & 1 & 1 & 6 & nil & nil & nil \\
\hline 12 & Dipterocarpaceae & 5 & 8 & 41 & 7 & 14 & 47 \\
\hline 13 & Ebenaceae & 1 & 4 & 9 & 1 & 9 & 39 \\
\hline 14 & Elaeocarpaceae & 1 & 1 & 1 & nil & nil & nil \\
\hline 15 & Erythroxylaceae & 1 & 1 & 2 & nil & nil & nil \\
\hline 16 & Euphorbiaceae & 8 & 10 & 18 & 9 & 16 & 32 \\
\hline 17 & Fagaceae & 2 & 3 & 7 & 1 & 2 & 2 \\
\hline 18 & Flacourtiaceae & 2 & 2 & 3 & 3 & 3 & 7 \\
\hline 19 & Gnetaceae & nil & nil & nil & 1 & 1 & 1 \\
\hline 20 & Guttiferae & 4 & 12 & 33 & 2 & 9 & 27 \\
\hline 21 & Icacinaceae & 1 & 1 & 1 & nil & nil & nil \\
\hline 22 & Lauraceae & 7 & 7 & 24 & 5 & 5 & 11 \\
\hline 23 & Lecythidaceae & 1 & 2 & 2 & 1 & 3 & 19 \\
\hline 24 & Leguminosae & 6 & 6 & 18 & 5 & 6 & 13 \\
\hline 25 & Loganiaceae & 1 & 1 & 4 & nil & nil & nil \\
\hline 26 & Melastomataceae & 2 & 2 & 42 & 3 & 5 & 18 \\
\hline 27 & Meliaceae & 2 & 2 & 7 & 2 & 5 & 15 \\
\hline 28 & Moraceae & 3 & 4 & 13 & 4 & 4 & 36 \\
\hline 29 & Myristicaceae & 3 & 4 & 13 & 3 & 5 & 45 \\
\hline 30 & Myrsinaceae & 1 & 1 & 2 & 2 & 2 & 3 \\
\hline 31 & Myrtaceae & 1 & 12 & 28 & 3 & 15 & 46 \\
\hline 32 & Ochnaceae & 1 & 1 & 5 & 1 & 1 & 4 \\
\hline 33 & Olacaceae & 2 & 2 & 3 & nil & nil & nil \\
\hline 34 & Oxalidaceae & 1 & 1 & 1 & nil & nil & nil \\
\hline 35 & Pandaceae & nil & nil & nil & 1 & 1 & 1 \\
\hline 36 & Polygalaceae & 1 & 3 & 12 & 1 & 3 & 14 \\
\hline 37 & Rhizophoraceae & 2 & 2 & 18 & 1 & 1 & 9 \\
\hline 38 & Rosaceae & 1 & 2 & 2 & 1 & 1 & 1 \\
\hline 39 & Rubiaceae & 13 & 16 & 69 & 11 & 15 & 61 \\
\hline 40 & Rutaceae & 1 & 1 & 1 & nil & nil & nil \\
\hline 41 & Sapindaceae & 2 & 2 & 5 & 4 & 5 & 21 \\
\hline 42 & Sapotaceae & 4 & 5 & 11 & 3 & 4 & 15 \\
\hline 43 & Simaroubaceae & 1 & 1 & 1 & nil & nil & nil \\
\hline 44 & Sterculiaceae & 2 & 2 & 5 & 1 & 1 & 1 \\
\hline 45 & Thymelaeaceae & nil & nil & nil & 1 & 1 & 1 \\
\hline 46 & Theaceae & 2 & 3 & 5 & nil & nil & nil \\
\hline 47 & Tiliaceae & 1 & 1 & 1 & 1 & 1 & 3 \\
\hline 48 & Ulmaceae & 1 & 2 & 14 & 1 & 3 & 18 \\
\hline 49 & Verbenaceae & 1 & 1 & 19 & 2 & 2 & 7 \\
\hline \multirow[t]{2}{*}{50} & Violaceae & nil & nil & nil & 1 & 1 & 7 \\
\hline & Total & 109 & 154 & 516 & 104 & 177 & 636 \\
\hline
\end{tabular}


Table 2. The tree species composition based on the diameter class size at inland and riparian forest plot

\begin{tabular}{lccccc}
\hline \multirow{2}{*}{ Size class $(\mathrm{cm}) \mathrm{dbh}$} & \multicolumn{2}{c}{ Inland $(0.2 \mathrm{ha})$} & & \multicolumn{2}{c}{ Riparian $(0.2 \mathrm{ha})$} \\
\cline { 2 - 3 } \cline { 6 - 7 } & Ind & Ind/ha & & Ind & Ind/ha \\
\hline $1.00-4.99$ & 467 & 2335 & & 360 & 1800 \\
$5.00-9.99$ & 115 & 575 & & 84 & 420 \\
$10.0-14.99$ & 21 & 105 & & 36 & 180 \\
$>15.00$ & 39 & 195 & & 36 & 180 \\
\hline Total & 642 & 3210 & & 516 & 2580 \\
\hline
\end{tabular}

Table 3. Density of the five most dense tree species in inland and riparian forest of Chemerong Recreational Forest, Terengganu

\begin{tabular}{lllcc}
\hline Forest Types & Species & Family & Ind/ha & Coverage (\%) \\
\hline Riparian & Lijndenia laurina & Melastomataceae & 200 & 7.75 \\
$(\mathrm{n}=516$ ind) & Shorea macroptera & Dipterocarpaceae & 120 & 4.65 \\
& Gaertnera vaginans & Rubiaceae & 95 & 3.68 \\
Inland & Streblus elongatus & Moraceae & 150 & 4.67 \\
$(\mathrm{n}=636$ ind) & Gluta elegans & Anacardiaceae & 120 & 3.74 \\
& Mangifera caesia & Anacardiaceae & 110 & 3.43 \\
\hline
\end{tabular}

Table 4. Value of Importance $\left(S I V_{i}\right)$ of the species at both forest types in Chemerong Recreational Forest

\begin{tabular}{lllccrr}
\hline Forest Type & Spesies & Family & Rd (\%) & Rf (\%) & Rc (\%) & IVi (\%) \\
\hline Riparian & Lijndenia laurina & Melastomataceae & 7.75 & 1.66 & 4.85 & 5.22 \\
& Shorea macroptera & Dipterocarpaceae & 4.65 & 1.66 & 3.12 & 4.24 \\
& Gaertnera vaginans & Rubiaceae & 3.68 & 1.66 & 2.99 & 3.16 \\
& Vitex vestita & Verbenaceae & 3.68 & 1.66 & 3.21 & 2.27 \\
& Monocarpia marginalis & Annonaceae & 2.71 & 1.66 & 3.15 & 2.22 \\
Inland & Mangifera caesia & Anacardiaceae & 3.43 & 1.37 & 6.25 & 3.21 \\
& Streblus elongatus & Moraceae & 4.37 & 1.37 & 6.42 & 2.95 \\
& Gluta elegans & Anacardiaceae & 3.74 & 1.37 & 4.14 & 2.70 \\
& Mangifera griffithii & Anacardiaceae & 3.12 & 1.02 & 1.46 & 2.45 \\
& Swintonia floribunda & Anacardiaceae & 2.65 & 1.37 & 2.28 & 2.39 \\
\hline
\end{tabular}

Note: $\mathrm{Rd}$ - Relative density; Rc - Dominance; Rf - Relative frequency.

with 120 and $110 \mathrm{ind} /$ ha respectively (Table 3 ). This result was dissimilar to previous studies in Peninsular Malaysia where Euphorbiaceae was reported to have the highest density of the inland forest (Raffae 2003; Norwahidah, 2005; and Khairil et al., 2011) and riverine forest (Foo, 2005; Khairil et al., 2011; 2014a).

\section{Importance value index (IVi)}

Lijndenia laurina (Melastomataceae) was the most important species at the riparian forest with the SIVi of $5.22 \%$, followed by Shorea macroptera (Dipterocarpaceae) and Gaertnera vaginans with $4.24 \%$ and $3.16 \%$ respectively. In the inland forest, the trend was different as Mangifera caesia was the most important species with the SIVi of $3.21 \%$, followed by Streblus elongatus (Moraceae) and Gluta elegans (Anacardiaceae) with $2.95 \%$ and
$2.70 \%$ respectively (Table 4 ). The result observed in this study was different to Khairil et al. (2011; $2014 b$ ) as they found the important species at inland forest of Chini watershed was Endospermum diadenum (Euphorbiaceae) whereas Ganua motleyana (Sapotaceae) was the most important species in a riverine forest plot. According to Brower (1997), a species with SIVi of more than $10 \%$ can be considered as the dominant species in a particular community. Based on the results, all the species recorded from both forest types have IVi \% of less than $10 \%$, indicating that none of the species are dominant at Chemerong Recreational Forest.

\section{Species diversity and similarity}

The Species Diversity Index $\left(H^{\prime}\right)$ calculated for inland forest was 5.14 and was slightly higher than a riparian forest with $\mathrm{H}^{\prime}$ of 5.04. According 
to Magurran (2011), the value of H' usually lies between 1.5 and 3.5 , although in exceptional cases, the value can exceed 4.5. Therefore, the values of the diversity index in these two forest types were considered exceptionally high. In terms of Community similarity, we found the value of the Sorenson Community Coefficient $(C C s)$ index for both communities was low with the value of 0.38 . This indicated only $38 \%$ of the tree species occurring at both forest types are similar while the other $62 \%$ of the tree species between both forest types were dissimilar.

\section{Endemism and conservation status}

According to $\mathrm{Ng}$ et al. (1991) there were 2,830 plant species found to be endemic to Peninsular Malaysia while the total number of endemic trees is 746 species. The endemic tree species in this study represented $4.42 \%$ of endemic trees in Peninsular Malaysia, which consists of 33 species and 15 families (Table 5). In terms of conservation status of the tree species, at least 55 species in this study, equivalent to $20.7 \%$ of the total species recorded were listed in the International Union for Conservation of Nature (IUCN) Red Data Book in 2016 and Malayan Plant Red list (Chua et al., 2010) (Table 6).

\section{Soil characteristics}

Soils from both forest types were acidic with $\mathrm{pH}$ less than 6 (Table 7). This result was similar with other previous researches conducted in tropical forest of Malaysia by Paoli et al. (2008); Adzmi et al. (2010); Khairil et al. (2014a; 2014b) and Khairil and Burslem, (2018) where they also found that most of the soil pH was between 4-6 with high concentrations of $\mathrm{Al}^{3+}$. Mean available $\mathrm{P}$ was higher in the riparian forest compared to the inland forest while the mean of available $\mathrm{K}$ and $\mathrm{Mg}$, organic matter $(\mathrm{OM})$ and moisture content were slightly higher in the inland forest compared to riparian forest (Table 8). This result was similar to Khairil

Table 5. Endemic tree species in Peninsular Malaysia that can be found at Chemerong Recreational Forest, Terengganu, Peninsular Malaysia

\begin{tabular}{|c|c|c|}
\hline Family & Species & Location \\
\hline Annonaceae & Cyathocalyx pruniferus & KI, Tg, Pk, Ph, SI, MI, Jh \\
\hline Annonaceae & Popowia fusca & Prk, Ph, Sp \\
\hline Annonaceae & Xylopia magna & Ked, Kt, Tg, Prk, Pah, Sel, NS \\
\hline Annonaceae & Goniothalamus umbrosus & $\mathrm{Pn}, \mathrm{KI}, \mathrm{Tg}$ \\
\hline Apocynaceae & Kopsia macrophylla & $\mathrm{Kl}, \mathrm{Tg}, \mathrm{Pk}, \mathrm{Ph}, \mathrm{NS}, \mathrm{Ml}, \mathrm{Jh}$ \\
\hline Chrysobalanaceae & Atuna penangiana & Pen, KI, Tg, Pk, Jh \\
\hline Dilleniaceae & Dillenia reticulata & MI, Pah, Tg \\
\hline Ebenaceae & Diospyros ismailii & Ked, Tg, Ph, Sel, Joh \\
\hline Ebenaceae & Diospyros nutans & Kt, Prk, Ph, Sel, NS, MI \\
\hline Ebenaceae & Diospyros scortechinii & Kt, Tg, Prk, Ph, NS \\
\hline Ebenaceae & Diospyros argentea & Tg, Pk, Ph, SI, NS, MI, Jh \\
\hline Ebenaceae & Diospyros lanceifolia & Peninsular Malaysia \\
\hline Euphorbiaceae & Aporosa globifera & Ked, Pn, KI, Ph \\
\hline Euphorbiaceae & Aporosa nervosa & Peninsular Malaysia \\
\hline Euphorbiaceae & Croton erythrostachys & Tganu, Pah, Sel, NS, MI, Joh \\
\hline Flacourtiaceae & Casearia clarkei & Pen, Prk, Sel, Ml \\
\hline Flacourtiaceae & Scaphocalyx spathacea & Ktan, Pah, Sel, NS, MI, Joh \\
\hline Guttiferae & Garcinia opaca & Prk, Pah, Sel \\
\hline Lauraceae & Alseodaphne sp. 1 & Jh, Tg \\
\hline Lauraceae & Litsea spathacea & $\mathrm{Pn}, \mathrm{KI}, \mathrm{Pk}, \mathrm{Ph}, \mathrm{SI}$ \\
\hline Lauraceae & Beilschmiedia insignis & $\mathrm{KI}, \mathrm{Pk}, \mathrm{Ph}, \mathrm{Sl}$ \\
\hline Lauraceae & Cinnamomum mollissimum & Pen, Kt, Tg, Prk, Ph, Sel \\
\hline Myrtaceae & Syzygium conglomeratum & SI, MI, Jh, Sp \\
\hline Oxalidaceae & Sarcotheca monophylla & $\mathrm{Pk}, \mathrm{Ph}, \mathrm{Sl}, \mathrm{Ml}$ \\
\hline Rubiaceae & Saprosma scortechinii & $\mathrm{Kd}, \mathrm{Tg}, \mathrm{Pk}, \mathrm{Ph}$ \\
\hline Rubiaceae & Psydrax maingayi & Tg, Ph, Prk, Sel, NS, MI, Joh \\
\hline Rubiaceae & Morinda corneri & $\mathrm{Tg}, \mathrm{Ph}$ \\
\hline Rubiaceae & Gaertnera obesa & Ml, Jh. Sp \\
\hline Rubiaceae & Urophyllum ferrugineum & Pk, Jh \\
\hline Rubiaceae & Psychotria griffithii & Tg, Pk, SI, NS, Ml, Jh \\
\hline Sapindaceae & Trigonachras sp. 1 & $\operatorname{Tg}$ \\
\hline Sapotaceae & Madhuca tubulosa & Tg, Jh \\
\hline Theaceae & Adinandra maculosa & $\mathrm{Ph}, \mathrm{Kl}, \mathrm{Tg}, \mathrm{Pk}, \mathrm{SI}, \mathrm{Ph}$ \\
\hline
\end{tabular}

Notes: $\mathrm{Tg}=$ Terengganu; $\mathrm{Pk}=$ Perak; $\mathrm{Jh}=$ Johor; $\mathrm{KI}=$ Kelantan; $\mathrm{SI}=$ Selangor; $\mathrm{Ph}=\mathrm{Pahang} ; \mathrm{MI}=$ Melaka; Sel = Selangor; NS = Negeri Sembilan; Ked = Kedah; Sp = Singapore; $\mathrm{Pen}=$ Penang; $\mathrm{Ph}=\mathrm{Pahang}$ 
Table 6. The species status which require conservation based on Red Data Book (IUCN 2016)

\begin{tabular}{|c|c|c|c|}
\hline No & Species & Family & Conservation Status \\
\hline 1 & Aglaia crassinervia & Meliaceae & Lower Risk/near threatened \\
\hline 2 & Aglaia forbesii & Meliaceae & Lower Risk/least concern \\
\hline 3 & Aglaia malaccensis & Meliaceae & Lower Risk/near threatened \\
\hline 4 & Aglaia odoratissima & Meliaceae & Lower Risk/least concern \\
\hline 5 & Amesiodendron chinense & Sapindaceae & Lower Risk/near threatened \\
\hline 6 & Anisophyllea corneri & Anisophylleaceae & Lower Risk/least concern \\
\hline 7 & Anisophyllea disticha & Anisophylleaceae & Lower Risk/least concern \\
\hline 8 & Anisoptera laevis. & Dipterocarpaceae & Endangered \\
\hline 9 & Aquilaria malaccensis & Thymelaeaceae & Vulnerable \\
\hline 10 & Atuna penangiana & Chrysobalanaceae & Vulnerable \\
\hline 11 & Beilschmiedia insignis & Lauraceae & Lower Risk/least concern \\
\hline 12 & Bhesa paniculata & Celastraceae & Lower Risk/least concern \\
\hline 13 & Brackenridgea hookeri & Ochnaceae & Lower Risk/least concern \\
\hline 14 & Calophyllum soulattri. & Guttiferae & Lower Risk/least concern \\
\hline 15 & Canarium littorale & Burseraceae & Lower Risk/least concern \\
\hline 16 & Cotylelobium lanceolatum & Dipterocarpaceae & Vulnerable \\
\hline 17 & Cratoxylum arborescens & Guttiferae & Lower Risk/least concern \\
\hline 18 & Cyathocalyx pruniferus & Annonaceae & Lower Risk/least concern \\
\hline 19 & Dacryodes costata & Burseraceae & Lower Risk/least concern \\
\hline 20 & Dacryodes rostrata & Burseraceae & Lower Risk/least concern \\
\hline 21 & Diospyros argentea & Ebenaceae & Lower Risk/least concern \\
\hline 22 & Diospyros ismailii & Ebenaceae & Lower Risk/least concern \\
\hline 23 & Diospyros nutans & Ebenaceae & Lower Risk/least concern \\
\hline 24 & Diospyros scortechinii & Ebenaceae & Lower Risk/least concern \\
\hline 25 & Dipterocarpus crinitus & Dipterocarpaceae & Endangered \\
\hline 26 & Dipterocarpus grandiflorus & Dipterocarpaceae & Critically endangered \\
\hline 27 & Dipterocarpus oblongifolius & Dipterocarpaceae & Lower Risk \\
\hline 28 & Dyera costulata & Apocynaceae & Lower Risk/least concern \\
\hline 29 & Euonymus javanicus & Celastraceae & Lower Risk/least concern \\
\hline 30 & Garcinia opaca & Guttiferae & Lower Risk/least concern \\
\hline 31 & Gnetum gnemon & Gnetaceae & Least Concern \\
\hline 32 & Hopea griffithii & Dipterocarpaceae & Vulnerable \\
\hline 33 & Horsfieldia irya & Myristicaceae & Lower Risk/least concern \\
\hline 34 & Knema conferta & Myristicaceae & Lower Risk/least concern \\
\hline 35 & Koompassia malaccensis & Leguminosae & Lower Risk/conservation dependent \\
\hline 36 & Litsea spathacea & Lauraceae & Lower Risk/least concern \\
\hline 37 & Madhuca tubulosa & Sapotaceae & Lower Risk/conservation dependent \\
\hline 38 & Mangifera caesia & Anacardiaceae & Lower Risk/least concern \\
\hline 39 & Myristica cinnamomea & Myristicaceae & Lower Risk/least concern \\
\hline 40 & Ochanostachys amentacea & Olacaceae & Data Deficient \\
\hline 41 & Popowia fusca & Annonaceae & Lower Risk/least concern \\
\hline 42 & Prunus arborea var. arborea & Rosaceae & Lower Risk/least concern \\
\hline 43 & Prunus polystachya & Rosaceae & Lower Risk/least concern \\
\hline 44 & Santiria apiculata & Burseraceae & Lower Risk/least concern \\
\hline 45 & Santiria laevigata & Burseraceae & Lower Risk/least concern \\
\hline 46 & Sarcotheca monophylla & Oxalidaceae & Lower Risk/near threatened \\
\hline 47 & Scaphium macropodum & Sterculiaceae & Lower Risk/least concern \\
\hline 48 & Shorea curtisii ssp. curtisii & Dipterocarpaceae & Lower Risk/least concern \\
\hline 49 & Shorea guiso & Dipterocarpaceae & Critically Endangered \\
\hline 50 & Shorea leprosula & Dipterocarpaceae & Endangered \\
\hline 51 & Shorea macrantha & Dipterocarpaceae & Critically Endangered \\
\hline 52 & Shorea multiflora & Dipterocarpaceae & Lower Risk/least concern \\
\hline 53 & Vatica maingayi & Dipterocarpaceae & Critically endangered \\
\hline 54 & Vatica umbonata & Dipterocarpaceae & Lower Risk/least concern \\
\hline 55 & Xylopia magna & Annonaceae & Lower Risk/least concern \\
\hline
\end{tabular}


Table 7. The average chemical composition of soil and variance analysis for each forest type in Chemerong Recreational Fores, Terengganu, Peninsular Malaysia

\begin{tabular}{lccc}
\hline Soil content & Riparian forest & Inland forest & $P$ value \\
\hline $\mathrm{pH}$ & $4.04 \pm 0.23$ & $3.90 \pm 0.23$ & 0.188 \\
Available P (meq/100 g) & $2.91 \pm 1.13$ & $1.59 \pm 0.30$ & $0.002^{* *}$ \\
Available K (meq/100 g) & $100.95 \pm 35.05$ & $121.25 \pm 24.61$ & 0.626 \\
Available Mg (meq/100 g) & $26.46 \pm 11.06$ & $32.31 \pm 31.50$ & 0.587 \\
Electric conductivity $(\mathrm{dS} / \mathrm{m})$ & $19.21 \pm 0.24$ & $23.02 \pm 0.38$ & $0.017^{*}$ \\
Ammonium nitrogen & $5.55 \pm 2.25$ & $12.41 \pm 2.81$ & $0.000^{\star \star *}$ \\
Nitrate (NO3) (meq/100 g) & $6.85 \pm 5.26$ & $17.15 \pm 4.37$ & $0.000^{\star * *}$ \\
Cation exchange capacity (CEC) & $7.98 \pm 0.86$ & $8.12 \pm 0.64$ & 0.673 \\
Cation acid (meq/100 g) & $3.93 \pm 0.82$ & $3.75 \pm 0.57$ & 0.575 \\
Cation base (meq/100 g) & $4.05 \pm 0.26$ & $4.37 \pm 0.56$ & 0.111 \\
Moisture content $(\%)$ & $5.27 \pm 4.27$ & $8.91 \pm 2.11$ & $0.027^{*}$ \\
Organic matter $(\mathrm{OM})$ & $4.84 \pm 1.32$ & $7.77 \pm 1.48$ & $0.000^{\star * *}$ \\
\hline
\end{tabular}

Note: $p<0.05^{\star} ; p<0.005^{\star *} ; p<0.001^{*}$.

Table 8. Matrix correlation of soil content in Chemerong Recreational Forest

\begin{tabular}{|c|c|c|c|c|c|c|c|c|}
\hline & $\mathrm{pH}$ & OM & CEC & $\mathrm{Mg}$ & $\mathrm{K}$ & $\mathrm{P}$ & $\%$ Clay & $\%$ Silt \\
\hline OM & -0.442 & & & & & & & \\
\hline CEC & -0.221 & 0.164 & & & & & & \\
\hline $\mathrm{Mg}$ & -0.002 & -0.093 & -0.034 & & & & & \\
\hline $\mathrm{K}$ & 0.005 & -0.081 & -0.08 & $0.986^{\star \star *}$ & & & & \\
\hline $\mathrm{P}$ & 0.386 & -0.465 & -0.166 & -0.136 & -0.087 & & & \\
\hline$\%$ Clay & 0.379 & $-0.565^{\star *}$ & 0.019 & -0.23 & -0.211 & $0.709^{\star \star \star}$ & & \\
\hline$\%$ Silt & -0.023 & $0.574^{\star \star}$ & 0.045 & 0.128 & 0.123 & -0.369 & $-0.469^{*}$ & \\
\hline$\%$ Sand & -0.344 & 0.001 & -0.046 & 0.093 & 0.079 & -0.342 & -0.528 & $-0.501^{*}$ \\
\hline
\end{tabular}

Note: ${ }^{*} p<0.05,{ }^{* *} p<0.01,{ }^{* * *} p<0.001$.

et al. $(2014 \mathrm{a} ; 2014 \mathrm{~b})$ where they found the OM in inland forest was higher than in the seasonal flood and riverine forest. There are significant differences in available phosphorus $(\mathrm{P})(\mathrm{p}<0.01)$, nitrate $\left(\mathrm{NO}^{3-}\right)$ $(p<0.001)$, electric conductivity $(\mathrm{EC})(\mathrm{p}<0.05)$, organic matter $(\mathrm{OM})(\mathrm{p}<0.001)$ and moisture content $(p<0.05)$ between the two types of forests (Table 6). Throughout, the correlation between the physicochemical content of soil in this study was moderate. Available $\mathrm{P}$, for instance, had a positive correlation with the percentage of clay $(p<0.001)$ (Table 7$)$. This indicated the soil content, which had high available $\mathrm{P}$ may have a higher percentage of clay. There were also positive correlations between available $\mathrm{Mg}$ and available K $(\mathrm{p}<0.001)$, silt with $\mathrm{OM}(\mathrm{p}<0.01)$, but negative correlation was found between clay with silt $(\mathrm{p}<0.05)$, clay with $\mathrm{OM}(\mathrm{p}<0.01)$ and silt with sand $(p<0.05)$ (Table 6). Soil with high percentage of clay may have less percentage of silt and $\mathrm{OM}$.

\section{Soil-plant relationship}

The Canonical Correspondence Analysis (CCA) on the soil-plant relationship was conducted based on the selected 39 tree species (Table 9). The selection of the plant species was based on their occurrence within the subplots where the occurrences less than three were eliminated to ease the CCA following Barruch (2005). The eigenvalues for the first and second CCA axes had low values of 0.568 and 0.234 respectively Moreover, the total inertia observed in the CCA analysis was only 0.655 and only $56 \%$ of the variation was explained by the first axis, which suggests that the overall association between the species and environmental matrices was low. Based on the Monte-Carlo permutation test, there was no significant difference of the eigenvalues for the three ordination axes $(p>0.05)$. The percentage variance of the species environment relation given was cumulatively from the CCA, which can be obtained by weighted regression (Nizam et al., 2006; Khairil et al., 2014a; 2015) (Table 10). The inland and riparian forest tree group was not clearly separated on the CCA ordination diagram. Nevertheless the vector for soil available $\mathrm{P}, \mathrm{Mg}$ and $\mathrm{pH}$ were the longest among the soil variables, which suggests that these elements may have an important influence on species' distributions. The species preference in relations to environmental variables is illustrated in the speciesenvironment bi-plot in Figure 2. At least nine tree species had significant association with soil 
Table 9. The list of 39 out of 265 selected tree species in Canonical Correspondence Analysis (CCA) at Chemerong Recreational Forest, Terengganu, Malaysia

\begin{tabular}{clcl}
\hline Code & Species & Code & Species \\
\hline 1 & Anisophyllea disticha (Jack) Baill. & 21 & Knema laurina (Blume) Warb. var. laurina \\
2 & Anisophyllea scortechinii King & 22 & Lijndenia laurina Zoll \& Moritzi \\
3 & Artocarpus scortechinii King & 23 & Lindera lucida (Blume) Boerl. \\
4 & Baccaurea parviflora (Müll. Arg.) Müll. Arg. & 24 & Monocarpia marginalis (Scheff.) J. Sinclair \\
5 & Barringtonia scortechinii King & 25 & Ochanostachys amentacea Mast. \\
6 & Brackenridgea hookeri (Planch.) A. Gray & 26 & Palaquium rostratum (Miq.) Burck \\
7 & Calophyllum canum Hook.f. & 27 & Pellacalyx axillaris Korth. \\
8 & Canarium littorale Blume & 28 & Ryparosa wallichii Ridl. \\
9 & Ctenolophon parvifolius Oliv & 29 & Saprosma scortechinii King \& Gamble \\
10 & Cynometra malaccensis Meeuwen & 30 & Shorea guiso (Blanco) Blume \\
11 & Dacryodes rostrata (Blume) H.J. Lam & 31 & Shorea macrantha Brandis \\
12 & Diospyros buxifolia (Blume) Hiern & 32 & Shorea macroptera Dyer \\
13 & Dryobalanops oblongifolia Dyer ssp. & 33 & Streblus elongatus (Miq.) Corner \\
& occidentalis P.S. Ashton & 34 & Syzygium griffithii (Duthie) Merr. \& L.M. Perry \\
14 & Euonymus javanicus Blume & 35 & Vitex vestita Wall. ex Schauer \\
15 & Gaertnera obesa Hook.f. ex C.B. Clarke & 36 & Xanthophyllum affine Korth. ex Miq. \\
16 & Garcinia eugeniifolia Wall. ex T. Anderson & 37 & Xanthophyllum griffithii Hook.f. ex A.W. Benn. ssp. \\
17 & Garcinia nigrolineata Planch. ex T. Anderson & & erectum Meijden \\
18 & Gironniera nervosa Planch. & 38 & Xerospermum noronhianum (Blume) Blume \\
19 & Goniothalamus macrophyllus (Blume) & 39 & Xylopia ferruginea (Hook.f. \& Thomson) \\
& Hook.f. \& Thomson & & Hook.f. \& Thomson var. ferruginea \\
20 & Knema conferta (King) Warb. & & \\
\hline
\end{tabular}

Table 10. The summary of CCA vegetation analysis with the edaphic factor at Chemerong Recreational Forest

\begin{tabular}{lcccc}
\hline Axis & 1 & 2 & 3 & Total inertia \\
\hline Eigenvalues & 0.568 & 0.234 & 0.103 & 0.655 \\
Variance in species data & & & & \\
$\quad \%$ of variance explained & 56.5 & 33.2 & 11.6 & \\
Cumulative \% explained & 56.5 & 89.7 & 101.3 & \\
Pearson Correlation, Spp-Envt* & 0.973 & 0.968 & 0.985 & \\
Kendall (Rank) Corr., Spp-Envt & 0.667 & 0.667 & 1.000 & \\
\hline
\end{tabular}

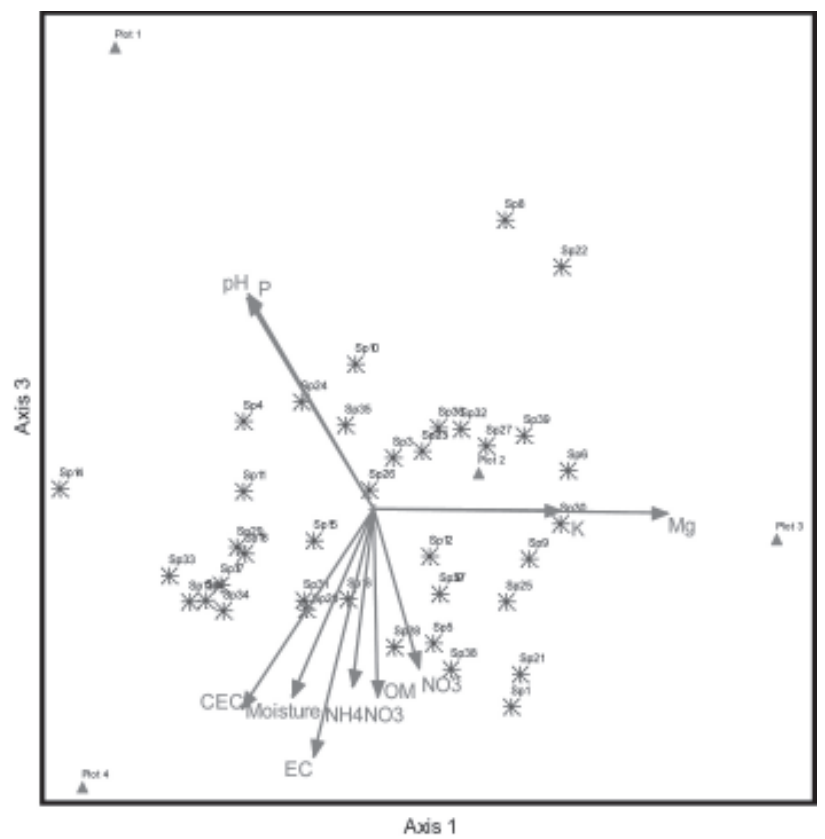

Fig. 2. CCA bi-plot for the species and variables of soil which show the relationship of tree species distribution with physicochemical of soil. 
Table 11. List of the species which were highly influenced by the physico-chemical of soil elements

\begin{tabular}{|c|c|c|c|c|c|}
\hline Code & Species & $\mathrm{n}$ & Habitat description & Family & Elements \\
\hline 5 & $\begin{array}{l}\text { Barringtonia } \\
\text { scortechinii }\end{array}$ & 9 & $\begin{array}{l}\text { In undisturbed coastal, swamp, and mixed dipterocarp } \\
\text { forests up to } 700(-1300) \mathrm{m} \text { altitude. Often on alluvial } \\
\text { sites or near rivers, but also on hillsides and ridges. } \\
\text { On sandy to clay soils, also on limestone. }\end{array}$ & Lecythidaceae & $\mathrm{NO}_{3}$ \\
\hline 16 & $\begin{array}{l}\text { Garcinia } \\
\text { eugeniifolia }\end{array}$ & 14 & Forest understorey. Lowland forest. & Cluisaceae & $\begin{array}{l}\text { Air dry } \\
\text { moisture }\end{array}$ \\
\hline 20 & $\begin{array}{l}\text { Knema } \\
\text { conferta. }\end{array}$ & 28 & $\begin{array}{l}\text { In undisturbed mixed dipterocarp, swamp and kerangas } \\
\text { forests up to } 600 \mathrm{~m} \text { altitude. On alluvial sites near or } \\
\text { along rivers and streams. On sandy soils. }\end{array}$ & Myristicaceae & CEC \\
\hline 24 & $\begin{array}{l}\text { Monocarpia } \\
\text { marginalis }\end{array}$ & 16 & $\begin{array}{l}\text { Undisturbed lowland forest up to } 1000 \mathrm{~m} \text { altitude. } \\
\text { Usually on hillsides and ridges with sandy soils. } \\
\text { In secondary forests usually present as a } \\
\text { pre-disturbance remnant. }\end{array}$ & Annonaceae & $\begin{array}{c}\text { Available } \\
\mathrm{P}, \mathrm{pH}\end{array}$ \\
\hline 6 & $\begin{array}{l}\text { Palaquium } \\
\text { rostratum }\end{array}$ & 17 & $\begin{array}{l}\text { In undisturbed mixed dipterocarp, kerangas, swamp, } \\
\text { coastal and sub-montane forests up to } 1200 \mathrm{~m} \\
\text { altitude. Growing both in alluvial sites as well as on } \\
\text { ridges, mostly on sandy soils, but also on clay and } \\
\text { limestone. }\end{array}$ & Sapotaceae & $\begin{array}{c}\text { Available } \\
\mathrm{P}, \mathrm{pH}\end{array}$ \\
\hline 8 & $\begin{array}{l}\text { Ryparosa } \\
\text { wallichii }\end{array}$ & 5 & $\begin{array}{l}\text { In undisturbed mixed dipterocarp forests up to } 800 \mathrm{~m} \\
\text { altitude. Usually on hillsides and ridges with clay to } \\
\text { sandy soils. In secondary forests usually present } \\
\text { as a pre-disturbance remnant tree. }\end{array}$ & Flacourtiaceae & OM, EC \\
\hline 30 & Shorea guiso & 4 & $\begin{array}{l}\text { In undisturbed forests up to } 400 \mathrm{~m} \text { altitude. Usually on } \\
\text { ridges with sandy and limestone soils. Scattered in } \\
\text { lowland forest on red soils, most common in slightly } \\
\text { seasonal climates. }\end{array}$ & Dipterocarpaceae & $\begin{array}{c}\text { Available } \\
\mathrm{K}, \mathrm{Mg}\end{array}$ \\
\hline 31 & $\begin{array}{l}\text { Shorea } \\
\text { macrantha }\end{array}$ & 4 & $\begin{array}{l}\text { In undisturbed mixed dipterocarp forests up to } 700 \mathrm{~m} \\
\text { altitude. On alluvial and dry sites (hillsides and ridges) } \\
\text { on clayey to sandy soils, also on limestone. }\end{array}$ & Dipterocarpaceae & CEC \\
\hline 38 & $\begin{array}{l}\text { Xerospermum } \\
\text { noronhianum }\end{array}$ & 17 & $\begin{array}{l}\text { In undisturbed mixed dipterocarp to sub-montane } \\
\text { forests up to } 1500 \mathrm{~m} \text { altitude. Mostly on hillsides and } \\
\text { alluvial sites with sandy to clay soils. }\end{array}$ & Sapindaceae & $\mathrm{NO}_{3}, \mathrm{OM}$ \\
\hline
\end{tabular}

Notes: $\mathrm{NO}_{3}$; Nitrate, CEC; cation exchange capacity, OM; organic matter, EC; electrical conductivity.

parameters, for instance by soil $\mathrm{pH}, \mathrm{EC}$, air dry moisture, available $\mathrm{Mg}, \mathrm{K}, \mathrm{P}$ and organic matter (OM). The list of species influenced by the edaphic factors is shown in Table 11.

Besides soil edaphic factor, other environmental factors should also be taken into consideration in future research such as altitude, topography, soil water content and forest gap (John et al., 2007; Baldeck et al., 2013; Khairil et al., 2014a; 2015) in investigating the factors shaping the pattern of the tree species diversity and composition in this area. Based on Whitmore, (1990); Itoh et al. (1995); John et al. (2007) and Baldeck et al. (2013), besides the physico-chemical characteristics of soil, sunlight, topography and altitude are among the important factors influencing the distribution of several tropical plant species.

\section{CONCLUSION}

Both riparian and inland forests in this study were significantly different in terms of species diversity and composition as well as their soil physicochemical contents. The soil edaphic factor has shown a less association with species composition in this study indicating the soil edaphic factor is not the main factor controlling the species distribution at this site. Further study is suggested to investigate other environmental factors that shape the pattern of the tree species diversity and composition in this area. Understanding the plant-soil relationship is of great importance to conserve and manage the forest ecosystems in the future. As a one of the important ecotourism sites in Terengganu, Malaysia, Chemerong Recreational Forest deserves 
attention from the stakeholders and state government to preserve this area and other reserve forests to ensure the natural green asset and other biotas in the state will still remain.

\section{ACKNOWLDEGMENTS}

We would like to thank Dr. Shamsul Khamis (UKM) and Mr. Razali bin Salam (UMT) for their help during plants identification process. This study was also funded by UniSZA Seed Money Grant UniSZA/ 11/GU (36).

\section{REFERENCES}

Ashton, P.S. 2008. Changing values of Malaysian forests: The challenge of biodiversity and its sustainable management.Journal of Tropical Forest Science, 20(4): 282-291.

Baldeck, C.A., Harms, K.E., Yavitt, J.B., John, R., Turner, B.L., Navarrete, H. \& Thomas, D.W. 2013. Soil resources and topography shape local tree community structure in tropical forests. Royal Society Publishing, 280: 201-225.

Baruch, Z. 2005. Vegetation-Environment Relationships And Classification of The Seasonal Savannas In Venezuela. Functional Ecology of Plants, 200(1): 49-64.

Black, C.A. 1968. Soil-Plant Relationship. New York: John Wiley And Sons, Inc.

Brower, J.E., Zar, J.H. \& Ende, C.N. 1997. Field and Labratory Methods For General Ecology. Fourth edition. Mc Graw Hill. Boston: 221-230.

Chua, L.S.L., Suhaida, M., Hamidah, M. \& Saw, L.G. 2010. Malaysia Plant Red List: Peninsular Malaysian Dipterocarpaceae. Forest Research Institute Malaysia (FRIM) \& Ministry of Natural Resources and Environment Malaysia, Malaysia. pp. 73, 146.

Condit, R., Ashton, P.S., Manokaran, N., LaFrankie, J.V., Hubbell, S.P. \& Foster, R.B. 1999. Dynamics of the forest communities at Pasoh and Barro Colorado: comparing two 50-ha plots. Philosophical Transactions of the Royal Society of London. Series B, Biological Sciences, 354 (1391): 1739-1748.

Davies, S.J., Tan, S., Lafrankie, J.V. \& Potts, M.D. 2005. Soil-related floristic variation in a hyperdiverse dipterocarp forest. In Ecological Studies: Pollination ecology and forest canopy (pp. 22-34).

De Deyn, G.B., Raaijmakers, C.E. \& Van Der Putten, W.H. 2004. Plant community development is affected by nutrients and soil biota. Journal of Ecology, 92(5): 824-834.
Foo, W.S. 2005. Comparative Studies on Tree Species Composition, Diversity and Above Ground Biomass at Riparian and Inland Forest, Kenong Forest Park, Pahang.

Forestry Department of Malaysia. 2008. Laporan Tahunan 2007. Kuala Lumpur.

Forestry Department of Malaysia. 2016. Laporan Tahunan 2015. Kuala Lumpur.

Itoh, A., Yamakura, T., Ogino, K., Lee, H.S. \& Ashton, P.S. 1995. Relationships between topography and distributions of two emergent species, Dryobalanops aromatica and $D$. lanceolata (Dipterocarpaceae) in a tropical rain forest, Sarawak. In: Seng, L.H., Ashton, P.S. \& Ogino, K. (eds.). Long term ecological research of tropical rain forest in Sarawak. Ehime University.

John, R., Dalling, J.W., Harms, K.E., Yavitt, J.B., Stallard, R.F., Mirabello, M. \& Foster, R.B. 2007. Soil nutrients influence spatial distributions of tropical tree species. Proceedings of the National Academy of Sciences of the United States of America, 104(3): 864-9.

Katabuchi, M., Kurokawa, H., Davies, S.J., Tan, S., \& Nakashizuka, T. 2012. Soil resource availability shapes community trait structure in a species-rich dipterocarp forest. Journal of Ecology, 100(3): 643-651.

Khairil, M. \& Burslem, D.F.R.P. 2018. Controls on foliar aluminium accumulation among populations of the tropical shrub Melastoma malabathricum L. (Melastomataceae). Tree Physiology, 38(11): 1752-1760.

Khairil, M., Nashriyah, M., Norhayati, N., Amin, S., \& Fatihah, N. 2013. Tree species composition, diversity and above ground biomass of two forest types at Redang Island, Peninsula Malaysia. Walailak Journal of Science and Technology, 10(1): 77-90.

Khairil, M., Siti-Meriam, A., Nur Fatihah, H.N., Nashriyah, M., Razali, M.S. \& Noor Amalina, R. 2015. Association of edaphic factors with herbal plants abundance and density in a recreational forest, Terengganu, Peninsular Malaysia. Malaysian Applied Biology, 44(2): 33-43.

Khairil, M., Wan Juliana, W.A. \& Nizam, M.S. 2014a. Edaphic influences on tree species composition and community structure in a tropical watershed forest in Peninsular Malaysia. Journal of Tropical Forest Science, 26(2): 284-294.

Khairil, M., Wan Juliana, W.A. \& Nizam, M.S. 2014b. Soil Properties and Variation between Three Forest Types in Tropical Watershed Forest of Chini Lake, Peninsular Malaysia. Sains Malaysiana 43(11): 1635-1643. 
Khairil, M., Wan Juliana, W.A., Nizam, M.S. \& Faszly, R. 2011. Community structure and biomass of tree species at Chini watershed forest, Pekan, Pahang. Sains Malaysiana, 40(11): 1209-1221.

Magurran, A.E. 1988. Ecological Diversity And Its Measurement. London: Chapman And Hall.

Norwahidah, Z.A. 2005. Comparative Study of Tree Species Composition, Diversity and Biomass of Riparian Forest and AdEacent Inland Forest at Tasik Chini, Pahang. Master Degree Thesis, National University of Malaysia, Malaysia (unpublished).

Nizam, M.S., Norziana, J., Sahibin, A.R. \& Latiff, A. 2006. Edaphic relationship among tree species in the National Park at Merapoh, Pahang, Malaysia. Journal Biosains 17: 37-53.

Ng, F.S.P. (ed.) 1978. Tree Flora of Malaya. A manual for foresters, Vol. 3. Longman Malaysia Sdn. Bhd. Kuala Lumpur, p.339.

Ng, F.S.P. (ed.) 1989. Tree Flora of Malaya. A manual for foresters, Vol. 4. Longman Malaysia Sdn. Bhd. Kuala Lumpur, p. 549.

Ng, F.S.P., Low, C.M. \& Mat Asri, N.S. 1991. Endemic Tree of Malay Peninsula. Research Pamphlet, FRIM Kepong, Kuala Lumpur, 1991.

Paoli, G.D., Curran, L.M. \& Slik, J.W.F. 2008. Soil nutrients affect spatial patterns of above ground biomass and emergent tree density in southwestern Borneo. Oecologia, 155(2): 287 299.
Proctor, J., Anderson, J.M., Chai, P. \& Vallack, H.W. 1983. Ecological Studies in Four Contrasting Lowland Rain Forests in Gunung Mulu National Park, Sarawak: I. Forest Environment, Structure and Floristics. Journal of Ecology, 71(1): 237260.

Raffae, A. (2003). Tree Species Diversity, Biomass and Economic Value of 2.6 ha Plot in Langkawi Island. Master Degree Thesis, National University of Malaysia (unpublished).

Rowell, D.L. 1994. Soil Science: Method and Applications. Longman, London.

Shamshuddin, J. 1981. Asas Sains Tanah. Dewan Bahasa dan Pustaka, Kuala Lumpur.

Sukri, R.S., Wahab, R.A., Salim, K.A. \& Burslem, D.F.R.P. (2012). Habitat Associations and Community Structure of Dipterocarps in Response to Environment and Soil Conditions in Brunei Darussalam, Northwest Borneo. Biotropica, 44(5): 595-605.

United Nations Development Programme, UNDP. 2017. Mainstreaming of Biodiversity Conservation into River Management, 263.

Whitmore, T.C. 1990. An Introduction to Tropical Rain Forest. Clarendon Press and Oxford University Press. Oxford. United Kingdom.

Whitmore, T.C. (ed.) 1972. Tree Flora of Malaya. Vol. 1, Malayan Forest Records No. 26. Longman Malaysia Sdn. Bhd., Kuala Lumpur.

Whitmore, T.C. (ed.) 1973. Tree Flora of Malaya. Vol. 2, Malayan Forest Records No. 26. Longman Malaysia Sdn. Bhd., Kuala Lumpur. 
